\title{
A geographic automata model of residential mobility
}

\author{
Paul M Torrens \\ Department of Geography, Arizona State University, Tempe, AZ 85287-0104, USA; \\ e-mail: torrens@geosimulation.com
}

Received 1 July 2004; in revised form 26 May 2005; published online 6 February 2007

\begin{abstract}
In this paper is described a model of residential mobility, built to simulate individual households, their perception of and reaction to varying conditions across different scales of interaction, and their movements to occupy housing in a physical, social, and economic environment. The methodology underpinning the model is based on an automata core, which leverages the advantages it offers in terms of representing individual entities and their rule-based interactions. This methodology is extended, however, to incorporate geography-specific functionality, with advantages for the modeling of human systems. The applicability of the methodology is demonstrated through the development of a rich model of residential mobility, in which individual households interact with other households and real-estate infrastructure, dynamically in space and time, to form synthetic communities and artificial property submarkets. Use of the model for what-if experimentation is demonstrated with synthetic economic and sociodemographic simulation scenarios.
\end{abstract}

\section{Introduction}

Research relating to residential mobility abounds (for an overview, see Clark, 1982a; $\mathrm{Wu}$ and $\mathrm{Li}, 2004$ ) and work in this area has explored many facets of mobility. Topics that have been addressed include the reasons for relocation (Rossi, 1955; Wolpert, 1965; 1966), the process of housing search (Clark and Flowerdew, 1982), the various ways in which households evaluate properties (Speare et al, 1975), and wider-scale dynamics of property markets and submarkets (DiPasquale and Wheaton, 1996), to identify but a few. Modeling and simulation are integral to much of this work and a variety of modeling methodologies have been employed in these endeavors (see Clark, 1982b; Clark and Flowerdew, 1982).

In recent years cellular automata (CA) and agent automata have been employed in the modeling of residential dynamics, with success (Benenson and Torrens, 2004). Though they offer significant advantages for urban simulation, much room remains for advancing the methodology in social-science contexts (Epstein, 1999); this is particularly true with respect to human systems, for which agency is of relevance. In this paper I argue that a modeling scheme based on geographic automata (GA) can further extend the success of the automata approach and can offer several advantages for studying residential mobility through modeling and simulation. My argument is supported with demonstration of the methodology, and discussion of its use in the building of a model of residential mobility and application in simulation scenarios.

The paper is organized as follows. In section 2 the topic of modeling residential mobility with automata tools is introduced, and a justification and methodology for building GA is described. Following this, the use of the methodology to build a model of residential mobility is detailed in section 3. Experimentation with this model is described in section 4 , in the context of exploring residential location scenarios, ahead of concluding remarks in section 5 . 


\section{Methodology}

The methodology used to build the residential mobility model relies on an automata core, extended to accommodate geographic concerns. The resulting GA inherit modeling schemes from other automata-based methods, but add additional distinct functionality.

\subsection{Automata, cells, and agents}

Automata are processing devices, capable of holding state variables and altering those states on the basis of internal transition functions, and they are endowed with the ability to input and respond to state information from other automata devices. Taken together, collections of automata may be designed to interact in a massively parallel context to perform some collective action or to evolve in a system setting. Two classes of automata are popularly used in urban research: CA and agent automata. Later, I will argue in favor of a third class of automata tool, GA, which I believe to offer advantages in modeling residential mobility.

Basic automata $(A)$ such as Turing machines and finite state machines (Sipper, $1997)$ are usually specified with state variables $(S)$ to express automata conditions at specific points in time $(t)$, and rules $\left(R_{S}\right)$ that govern transitions between and to those states as time progresses; the rules are formulated as functions that accept state information input $(I)$ from other automata. The state-rule-input combination may take on spatial form, with connections representing spatial interaction possibilities, and almost always involves some temporal treatment:

$$
A \sim\left(S, R_{S}, I\right) ; \quad R_{S}: S_{t} \rightarrow S_{t+1} .
$$

CA ( $A_{\mathrm{C}}$ in formula (2) below) are an extension to this basic idea, adding cells as a bounding mechanism within which an automaton exists discretely. This simple addition extends the processing abilities of automata significantly; state information exchange between $\mathrm{CA}$ is considered within the context of neighborhoods $(N)$, which may be formulated as a local area of influence within a lattice of connected CA.

$$
A_{\mathrm{C}} \sim\left(S, R_{S}, I_{N}\right) ; \quad R_{S}: S_{t} \rightarrow S_{t+1} .
$$

CA may be thought of as pixels on a television screen, each pixel being endowed with the ability to change states independently and exchange that information with adjacent pixels by diffusion. A dynamic picture emerges as the pixels interact through this diffusion, observable to the onlooker.

Agent automata are also capable of processing information and of exchanging it with other agent automata. Different agent automata methodologies rely on this general scheme, ranging from individual-based models to agent-based models and multiagent systems (Box, 2001; Ferber, 1999; Kohler and Gumerman, 2001; Russell and Norvig, 1995; Stefansson, 1997; Tesfatsion, 2002; Woolridge and Jennings, 1995). Agent automata differ from CA cells in several respects, but predominantly the difference is one of interpretation; inspiration for agents comes from the study of humans, from artificial intelligence research for the most part. The characteristics and rules used to design agents usually refer to behavior. Agents are commonly specified in a heterogeneous fashion, and are endowed with proactive and goal-oriented behavior, perception, communication skills, the ability to adapt and retain memory of their actions, and functionality to allow them to evolve through space and time.

\subsection{Geographic automata}

There is room to extend the automata concept even further, however. This is particularly salient with respect to geographic functionality. I argue in favor of a fourth genre of automata to complement basic automata, $\mathrm{CA}$, and agent automata-GA-and 
my contention is that GA better lend themselves to the simulation of geographic dynamics. The computational scheme for GA, their formulation in software as GA systems, and their methodological foundation in geographic information science and complex adaptive systems are described elsewhere (Torrens and Benenson, 2005). I focus on their relevance to modeling residential mobility in this paper, and the advantages that they offer in that context.

Essentially, what I am doing, in devising GA, is stripping the automata methodology to its basic foundation. GA are considered as a tool for representing entities and their characteristics as dynamic processing devices, first and foremost. The methodology is built from this foundation, with geography as the main consideration. GA can accommodate $\mathrm{CA}$ as well as agent automata functionality. In addition, GA may serve as a mechanism for uniting the two approaches, and also may offer distinct functionality in their own right.

The general scheme for a geographic automaton $G$ is specified as follows:

$$
\begin{aligned}
& G \sim\left(K, S, R_{S}, R_{L}, L, I_{N}, R_{N}\right), \\
& R_{S}: S_{t} \rightarrow S_{t+1}, \\
& R_{L}: L_{t} \rightarrow L_{t+1}, \\
& R_{N}: N_{t} \rightarrow N_{t+1} .
\end{aligned}
$$

GA retain state $(S)$, input $(I)$, and state transition rules $\left(R_{S}\right)$ of general automata, CA, and agent automata. Additional functionality is added, however.

(a) Ontology of GA entities $(K)$ - the ontology is based on fixture in space and time. Fixed GA act in a CA-like manner; nonfixed GA are more akin to agent automata. GA can combine both space - time fixture and nonfixture, however.

(b) Movement rules $\left(R_{L}\right)-\mathrm{GA}$ are endowed with the ability to move through the spaces in which they reside, progressing through space using any form of movement. A dedicated movement rule governs this behavior, independent of general state transition rules.

(c) Georeferencing conventions $(L)$ - a flexible framework for expressing locations of modeled entities in space and time allows GA to be registered to (fixed and nonfixed) settings in which they may be situated. This referencing may take on any form-for example, on the basis of local action, action at a distance, direct, and indirect relationships in space and time - and may be conveniently derived from geographic information systems (GIS) and science.

(d) Neighborhood rules $\left(R_{N}\right)$-dynamically configurable and heterogeneous neighborhood transition rules are added, thereby allowing for the flexible definition of relationships between automata. Again, these rules are independent of state transition rules and not only do they allow for elastic, asymmetric, and action-at-a-distance neighborhoods, but the neighborhood itself may be tied to other conditions (and rules) in a model in such a way that the neighborhood, and the rules that drive its formation, may vary over space and time.

\subsubsection{GA as a distinct automata-based simulation tool}

The additional functionality that GA offer over other automata tools has advantages for the modeling of residential mobility. The ontology of GA entities, based on fixture in space and time, is basic but important, as it supports a fundamental distinction between mobile entities (migrating households, socioeconomic and ethnic groups) and fixed objects (real estate, property submarkets, cities), but also permits both to coexist and interact in space (a social space, an economic space, a demographic space, a search space, etc). This has important implications for the representation of residential 
systems, in which human-human and human-environment interactions are of paramount importance. Mobile and fixed households interact with each other and between and within fixed properties. These interactions are distinct, with fundamentally different behavior and considerations for humans and their buildings.

CA are good at handling infrastructure, in which state conditions are influenced in a dispersive fashion - urban blight gradually rippling from house to house on a street as depreciation in the visual character of neighboring houses influences the general feel of the street, and thus the sales price of other houses. Agent automata are good at handling humans, and in particular their decision and choice rules. However, the phenomena also exist in a symbiotic relationship that cannot be adequately captured under a solely cellular-based or agent-based approach. Moreover, space is often an essential unifying and explanatory mechanism.

GA are based on an automata core, and share some characteristics with basic automata, cellular automata, and agent automata. The addition of geography is distinct, however, and has important implications for modeling residential mobility. It is worth discussing these distinctions before describing the use of the methodology for modeling residential mobility scenarios.

\subsubsection{The methodological importance of geography}

The main distinction between GA and CA or agent automata is the addition of geography to the general automata scheme. CA and agent automata are already endowed with geographic properties (lattice position, neighborhoods, state transition mediated through neighborhood input, and movement, in the case of mobile agent models). GA build upon these schemes, and allow for a greater degree of flexibility in the representation of geography. Delineation of GA does not need to be restricted to cell pixels and interaction is not necessarily limited to a neighborhood window. Similarly, GA may be agent based or not, or may offer attributes of both specifications within the same model. The GA framework is not restricted to a cellular-based or agent-based view of the world, but can become so if required. The explicit and flexible treatment of geography is particularly important in representing residential mobility.

GA allow for the representation of a variety of spatial structures. GA can exist in a spatial environment or not. They may be defined in a cellular fashion. These automata may take on a regular or irregular form, and taken together they may exist in a tessellated space, a network space, or in a spatial field. They might also be nonspatial, but designed to become spatial if and when necessary.

A variety of spatial processes may be accommodated: random perturbation of automata states in diverse locations within a lattice structure, true movement in a vector space, local-scale diffusion within a neighborhood of influence, action at a distance by direct and indirect means, or inertia. The process may be based on interaction in space or time or not. These processes might operate between fixed automata, between mobile automata, or between both.

GA may be global and local, or may be designed to operate in space and time across scales. This permits action at a distance or action at close proximity, both in space and time, and considered in direct and indirect contexts.

In addition, GA hold other advantages. They relate well, on an operational level, to object-oriented paradigms for building modeling software; they also interface relatively seamlessly with raster-based and vector-based GIS. Similarly, relationship conventions match well with object-oriented database-management systems and entity-relationship models (Benenson and Torrens, 2005; Benenson et al, 2005; Torrens and Benenson, 2005). Moreover, GA relate closely to complex adaptive systems in the consideration 
of their interactive dynamics. The flexibility of the approach allows for the nesting of entities and their relationships across scales, and facilitates the emergence of novel spatial ensembles across those boundaries (Torrens and Benenson, 2005).

\subsubsection{GA versus $C A$ and agent automata}

The use of CA in residential-mobility modeling follows pioneering work by Sakoda and Schelling in the 1970s (Sakoda, 1971; Schelling, 1978), who modeled sociospatial segregation in residential location behavior with the use of basic checkerboard models. CA models of local-scale relocation have also been developed by Batty (2001) and Schweitzer (2003). O'Sullivan's (2002) CA model of gentrification is also relevant. Several agent-based models of residential location behavior have also been built. Agents have been used in this context to construct multiagent systems composed of relocating households. Their behavior has been formulated on the basis of cultural and cognitive dissonance among individual households and the resulting patterns of segregation they influence. The simulations have been used to explore scenarios for the sociospatial segregation of diverse groups in Israeli cities (Benenson, 1998; Benenson et al, 2002), and to examine the properties of complex adaptive systems in those cities (Portugali et al, 1994; 1997). These CA and agent models may be differentiated from GA in a number of ways, considering the definition of cell geography, states, state transition rules, representation of agency, georeferencing, neighborhoods, and movement.

Cell geography is commonly defined in an arbitrary manner, as a function of the methodology rather than theory, in CA models of residential dynamics. Cells are pixellike, same sized, and uniform in spatial configuration, for example, in models by Sakoda (1971) and Schelling (1969; 1971; 1974; 1978). The models are formed in a basic checkerboard fashion, without consideration of real urban land parcels. The same is true of other CA models developed by Batty (2001) and Schweitzer (2003). These models were designed to function as simply as possible, however, and work very well pedagogically. O'Sullivan's (2002) graph-based CA models of gentrification characterize cells in terms of network nodes, whereas the residential CA models of Benenson and colleagues (2002) represent cells as irregular polygons. The GA scheme accommodates each of these forms of cellular geography. It requires only that entities be represented as automata and is indifferent to their geography. In addition, automata may be specified on the basis of entity-relationship schemes (Torrens and Benenson, 2005), following the entity-relationship model (Peckham et al, 1995).

CA models of residential dynamics have also treated cell-state characteristics simply, with households represented as simple black-and-white states with a binary contentment state, for example, in the Sakoda (1971) and Schelling (1969; 1971; 1974; 1978) schemes. The intention, in those models, is to abstract characteristics of households that might help to explain sociospatial segregation. There is no distinction between households and properties. O'Sullivan's (2002) work on gentrification does make this distinction; cells are used to simultaneously hold state characteristics for households and their homes in his example. These states are still mapped to the cell, however. GA may also accommodate those state schemes. Indeed, they have been found to be compatible with all urban automata models published in the literature (for a detailed list and description, see Torrens and Benenson, 2005). The advantage of GA, with respect to state descriptors, lies in the relationship between GA states and the other unique properties of the methodology_ontology, movement, neighborhood rules, and georeferencing. States can thus be expressed and interpreted explicitly through geography. For example, states of a household can be clearly distinguished from those of a home. Moreover, the set of those state descriptors for individual 
household members may take on heterogeneous form on an independent basis, or as they move through space and time relative to other simulated entities and objects.

Transition rules in CA models of residential mobility relate to state change. These are formulated in a variety of ways. Segregation models tend to incorporate rules designed on the basis of factors that alter a household contentment state in a cell, on the basis of the proportion of various social and ethnic mixes in a fixed neighborhood of cells around a cellular automaton, with this used as the input to a tolerance calculation. Other CA models rely on a rent-gap calculation as a transition rule (O’Sullivan, 2002). GA provide the means for connecting cell-like state transition with agent-like state transition, intuitively, through geographic considerations. As automata-based householders' income grows, for example, they may make capital improvements in their home. Deterioration in the condition of a neighboring structure, or an alteration in its land use, may prompt an increase in the dissatisfaction of a household that resides next door.

Representation of agency is quite cursory in many CA models of residential dynamics. Relationships between cell-based households are expressed in terms of contentment regarding collocation in local spaces in segregation models (Sakoda, 1971; Schelling, 1971). Once again, these models are designed to be simple. Recently, agent-based models based on cognitive dissonance have been developed by Benenson and colleagues, which provide a richer representation of agency, formulated on agent utility regarding the social and economic environment around them. The advantage of the GA approach lies in its ability to interpret agency through geography. GA can be made to focus on the exercising and differential application of decision and choice rules as a function of movement and varying conditions encountered in diverse geographies. This is particularly important in residential contexts - for example, in housing-search behavior.

CA models are incapable of representing true movement. Cells are fixed in a lattice. Unlike households, they do not uproot and move. Movement rules are absent in CA. The only form of movement that may take place does so by proxy means; state information is exchanged within a fixed neighborhood. In this sense states can diffuse through the system, iteration by iteration. However, action at distance (such as migration) is not easily facilitated in CA (Batty, 1997). Similarly, true vector motion with associated directions and velocities is also not possible in a CA context; this can be mimicked, as with Game-of-Life gliders (Gardner, 1970; 1971), through state exchange, but the movement is a function of the visual interpretation of graphic output of the models in these cases (Faith, 1998), rather than truly simulating movement or migration as we would understand it to operate in the real world. CA models get around this problem by representing movement in a proxy fashion. CA cells are randomly switched on or off in the Sakoda and Schelling models, and are activated with state conditions of black or white, content or malcontent, within a lattice of such cells. This gives the illusion of migration, with the result that cells are made to form globally distinct spatial patterns of segregation under simulation (figure 1). A similar mechanism is employed in graph-based CA. Agent automata do support movement, and there are many examples in the pedestrian and vehicle traffic literature (Torrens, 2005). Batty (2001) and Schweitzer (2003) have built agent-based models of city-scale residential mobility, with a focus on the movement of agents as particles under the influence of attraction surfaces, with that attraction determined by agglomeration factors. As mentioned, GA come equipped with dedicated movement rules, which are used to animate GA through locations. These rules are distinct from state transition rules and are designed to accommodate a rich range of movements relevant to residential location behavior: how households navigate, search, stay put, and are mobilized in modeled spaces. 

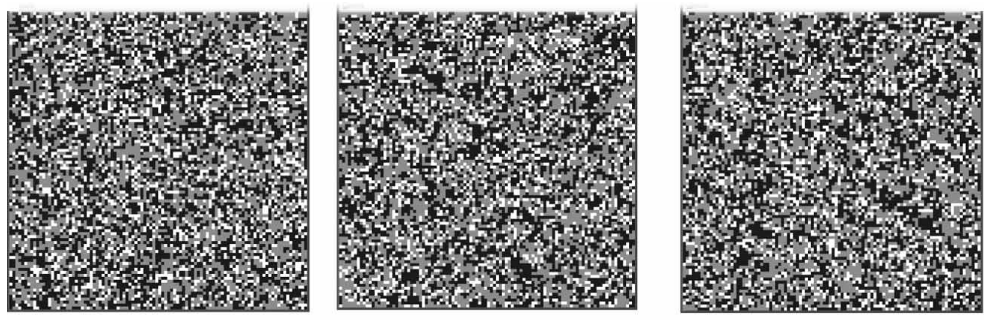

(a)
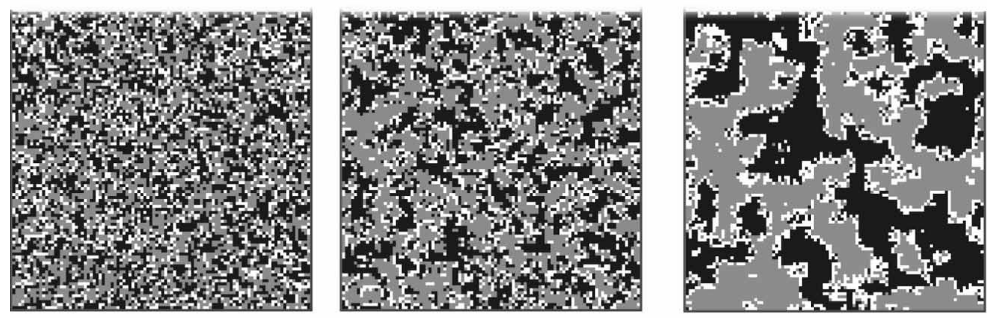

(b)
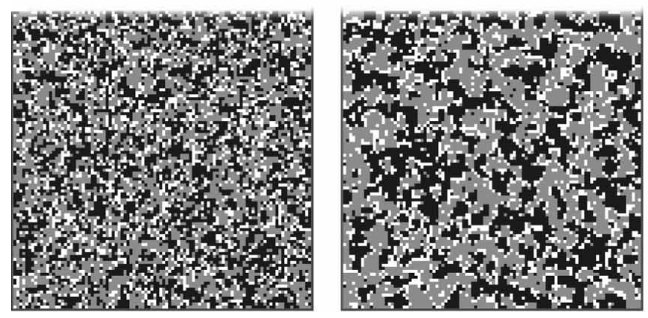

(c)

$t=1$

$t=5$

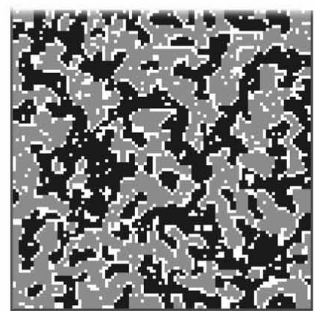

$t=50$

Figure 1. Automata implementation of the Schelling model, run with varying parameters: (a) 8000 households with 10\% tolerance, (b) 8000 households with $30 \%$ tolerance, (c) 8000 households with $40 \%$ tolerance. (Agents are black or gray; free space is white; $t$ denotes the number of iterations of the simulation.)

A range of residential-relevant movements can thus be represented, including entry and exit from property markets, housing-search behavior, the moving in and moving out of individual properties, geographical inertia, and migration dynamics.

Georeferencing in CA models relates to the positioning of cells in a regular, static, and uniform lattice structure. Referencing is accomplished by direct means alone. Cells and neighborhoods of adjacency are referenced to fixed cells. Georeferencing is extended in the GA methodology, and incorporates these conventions, but also allows for the expression of location conventions for automata relative to other automata or objects in space and time. Both direct and indirect georeferencing is accommodated. Direct georeferencing ties automata to a particular place at a particular time. Indirect georeferencing conventions relate automata to other objects in a simulated environment; they serve as pointers that express the position of automata relative to other entities in space-time. Those entities may themselves be fixed in space or not, and the conventions may take on a dynamic or static nature. In the context of residential dynamics, households may thus be registered to a home, a community, a property submarket (or all of those things), as well as being linked to households with which they interact as neighbors, buyers, sellers, etc. 
The fixture of location in the CA scheme affects CA neighborhoods. Neighborhoods of interaction are more commonly symmetric in form in CA approaches and are usually static in configuration, although some exceptions exist (see Shi and Pang, 2000). By contrast, GA are endowed with flexible and dedicated rulesets for determining relationships and for allowing those relationships to be varied in space and time. Neighborhood configurations are allowed to be defined flexibly, as networks, polygons, graphs, etc (Torrens and Benenson, 2005). The configurations and their determinants may also change over space and time. This flexibility is important in the context of residential mobility. Households may maintain a variety of neighborhood relationships simultaneously: social relationships between members of a household, next-door relationships with neighbors in proximal apartments or houses, buyer-seller relationships in property transactions, social affiliations with submarket residents of a given ethnic background, etc. Similarly, properties may maintain neighborhood relationships, with households, and also with other properties - houses on a real estate broker's listing, units in an apartment building, architecture consistencies with homes on a street, etc. Moreover, these relationships may well be interrelated and interdependent, and will most likely vary in space and time in a dynamic fashion as states and conditions change and evolve. The search space for a relocation event may change as a function of the properties that are evaluated, for example, with the result that the neighborhood window shrinks and stretches as the household's (and/or the submarket's) conditions evolve.

This flexibility can be captured through the use of neighborhood rules in GA contexts, rather than by working with fixed neighborhood windows defined a priori. For example, the neighborhood of consideration for a relocating household may be related to the information provided to it, its state in the life cycle, its income, social biases, etc. Moreover, the rule may be time dependent, with the result that the household's neighborhood of search narrows with the time spent searching, or the longer their home stays on the market unsold. It may also be geography dependent, with a search ignoring certain (over-budget, under-budget) submarkets in a city altogether, or concentrating around properties in a desirable school district or within transit commutes of an employment center.

\section{A GA model of residential mobility}

I will discuss the construction of a residential mobility model, composed of GA, in this section, to elaborate the GA methodology presented in section 2 and to emphasize the validity of the approach with respect to the representation and examination of residential systems. The model is used in section 4 to build simulation scenarios relating to residential mobility in a synthetic community of households and a hypothetical property submarket. The model is designed to represent households and properties in a realistic fashion, with life-like behaviors and characteristics. Much of the theoretical literature on residential mobility has been considered in designing the model: hierarchy in housing search (Speare et al, 1975), stress and resistance to mobility (Wolpert, 1966), household life cycles (Clark and Huang, 2003; Kendig, 1984; Pickvance, 1973), and household preferences for submarkets and real estate (DiPasquale and Wheaton, 1996; Waddell, 2000).

Residential mobility is treated as a two-stage process. First, households decide whether to initiate a relocation event or not, looking both to internal and to external stressors in formulating this decision. If they decide to engage in a housing search, they do so based on preferences that relate to their internal characteristics and those of the larger community and submarket in which the search is focused. Communities and submarkets are represented as comprising many individual properties and 
independent households (figure 2). The community and submarket are, however, treated simultaneously as an independent entity in the model, with the capacity to evolve within it and to serve as an object to which households react and with (and within) which they may interact.

The model is specified as a collection of interacting GA. Each automaton is designed, in a heterogeneous fashion, with reference to an entity ontology based on fixture in space, state variables relevant to that ontology, state transition rules, georeferencing conventions, movement rules, neighborhoods, and neighborhood rules. In addition, a set of constraint parameters is introduced to facilitate the generation of simulation runs.

\subsection{Entity ontology}

Spatial fixture-whether entities move or are in situ-is used as the basic ontology for defining entities in the model. A number of entities relevant to residential mobility are introduced under this umbrella: real estate submarkets, communities of residents, individual properties, resident households, and relocating households (figure 2). Relationships between those entities are hierarchical, such that the entities are nested spatially [figure 2(a)]. This mirrors Speare et al's (1975) taxonomy of factors governing residential mobility. It also introduces geography at a fundamental level. Real estate submarkets - which are widely regarded as being of importance in determining individual-level mobility (van der Vlist et al, 2002)-comprise properties that fall within their boundaries; both remain fixed in space. Properties contain resident households, which may be content to stay where they are, or may seek to relocate [Brown and Moore's (1970) movers and stayers]. These households form a dynamic community of people within the submarket. They are distinct and are treated as such in terms of their geography - they may move, for example; relocating households may actively engage in a property search within the submarket, although they may not necessarily belong to that community as residents.

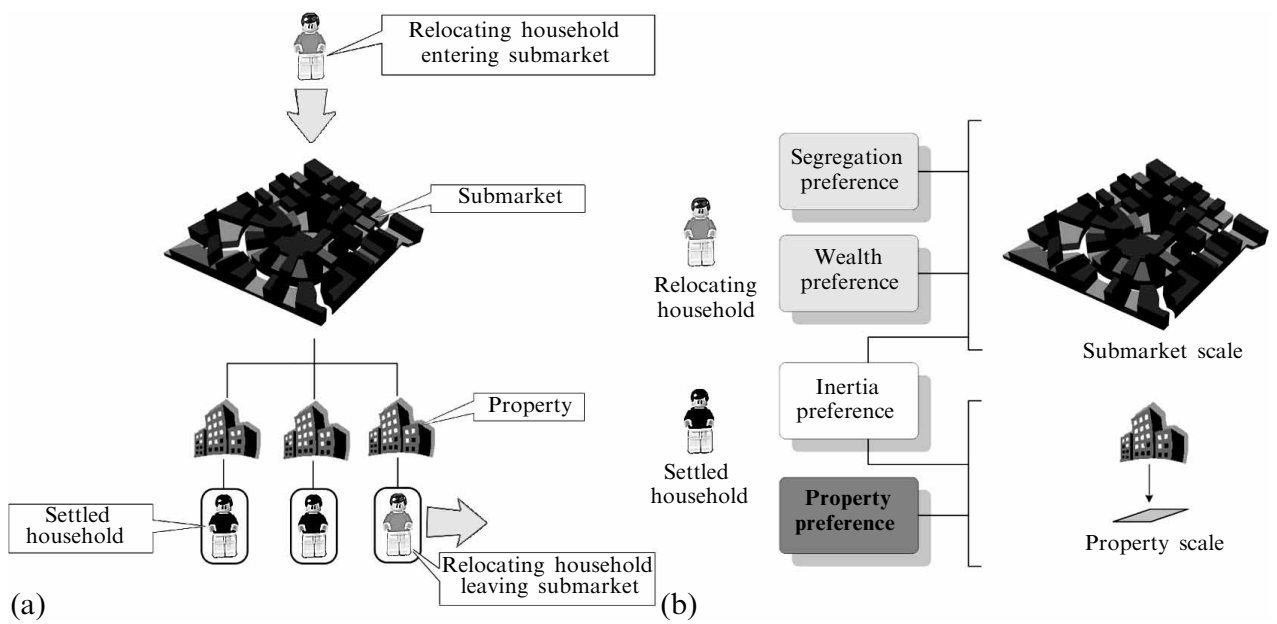

Figure 2. (a) The hierarchy of entities in the residential location model; (b) the organization of preferences across scales.

\subsection{State variables}

State variables are used to ascribe relevant characteristics to modeled entities, at various scales. They determine the behavior of modeled entities upon input to transition rules. Modeled households are heterogeneous with respect to these states. (For example, individual households are parameterized heterogeneously in the simulations 
introduced later, as illustrated in table 5 toward the end of the paper.) The nested hierarchy of entities enables units at higher scales to derive state conditions by means of dynamically collecting the attributes of entities within them, which may also be active at lower scales of observation. For example, average household income for a community may be calculated simply as the average income of households within it.

The GA employed in this model are used to represent 'atomic' (people-scale) spatially nonmodifiable entities and are attributed heterogeneous life-like states (and independent behaviors) that closely match the characteristics and functionality of their real-world counterparts (table 1). As the makeup of a community of those automata alters at an individual level, those changes register in state values at higher scales. Once again, these higher level entities are themselves represented as GA (table 1). Submarkets are fixed GA in the contexts of the examples discussed in this paper. They could, however, be treated as geographically dynamic GA, with their boundaries expanding or contracting as a function of the activities and interactions of the separately defined GA entities within them.

Individual property automata are used to represent the urban residential fabric. They are specified with an array of authentic state variables that lend them property-like attributes. The intention is to provide a realistic opportunity setting for household automata. Considered collectively, this setting forms a spatial landscape of socioeconomic attributes (after Knox, 1989; O’Flaherty, 1996; Rossi, 1955), with which simulated

Table 1. State variables used in the models.

\section{Residential submarkets}

Average property value

Maximum property value

Minimum property value

\section{Residential communities}

Total number of households

Total number of yellow households

Totol number of blue households

Total number of red households

Average household income

Average household age

\section{Fixed property automata}

Housing type (house, apartment)

Occupation status (occupied, not occupied)

Housing tenure (rent, own)

Sale or rental status (for sale, not for sale, for rent, not for rent, under offer)

Monthly mortgage value or rental value

Land use (residential, nonresidential)

Lot size

Density

Number of bedrooms

Time on the market

\section{Mobile household automata}

Household type (settled, relocating)

Average monthly household income

Average household age

Number of children

Household size

Ethnicity (yellow, blue, red)

Life-cycle stage (young, middle, senior)

Period of residency, in time steps 
households may engage and within which they may interact-location stressors (after Clark and Cadwaller, 1973); economic attributes (after DiPasquale and Wheaton, 1996; Rosen, 1974); and ethnic factors (after Cronin, 1982; Farley and Frey, 1994; Farley et al, 1978; Galster, 1991).

Similarly, household automata are specified with various state descriptors that help in informing their mobility behavior and in facilitating simulation of their awareness of the dynamic conditions that surround them. The set of state variables included in the model enables the specification of realistic household profiles, simply by combining different attributes. A proxy variable is used to represent ethnicity $(e)$, with households represented as either red, yellow, or blue automata. Following the discussion of life courses in the literature (Clark and Huang, 2003; Kendig, 1984; Pickvance, 1973), households are classified into particular life-cycle states $(l)$. This is a simplified interpretation of the life-cycle notion, but one that could be extended in principle. 'Young' households represent those that have recently left a family household unit and are striking out on their own for the first time. 'Middle' households are used to represent households that might be starting their own families or may have already started a family. 'Senior' households correspond to those households that are entering (or have already entered) retirement. Life-cycle stages are calculated on the basis of the average age of a household $(a)$ :

$$
\begin{array}{ll}
\text { if } 22 \leqslant a \leqslant 35, & l=\text { 'young'; } \\
\text { if } 35<a<65, & l=\text { 'middle'; } \\
\text { if } a \geqslant 65, & l=\text { 'senior'. }
\end{array}
$$

(Households with average ages below 22 are not considered in the model.)

\subsection{State transition rules}

State variables provide the ingredients for transition rules, as mentioned. Transition between these states is governed by two sets of rules in the model: those relating to transition in property, and those that are relevant to households.

Property state transition rules. The issue of devoting state transition rules to property entities is interesting. Transition rules are generally understood to act as mechanisms for assigning behaviors (in an agent-based context) or state transition functionality (in a CA context) to automata. In the real world, properties have very few actual behaviors of their own; they deteriorate if they are not maintained (O'Flaherty, 1996), but that is about it. Any functionality that they might be understood to offer relates to the contribution of real estate to a larger submarket for the most part, or the uses that properties afford residents. Submarket dynamics are more appropriately modeled at a submarket scale and household utility for property is perhaps better considered in terms of household preferences for real estate or the perception of its use (Golledge and Stimson, 1997; Rosen, 1974). To accommodate this in the model, properties are treated as containers for households, and households react to the characteristics of properties, among other stimuli.

State transition of property units is mediated directly by the households that interact with them, with the aforementioned considerations taken into account. Occupation status, tenure, sale or rental status, mortgage or rental value, and density states are altered directly by household automata as they examine, rent, buy, sell, and reside within the properties.

Only two state transition rules are assigned directly to property automata in the model. The first simply keeps track of the duration which a property that is for rent or sale spends on the market. The second adjusts the rental or sale price according to this state. If a property has spent too many days on the market $(m)$ without selling or 
renting - above a certain threshold of days $(\theta)$ - then its mortgage or rental value $(v)$ is discounted by a proportional value $(\lambda)$ in a subsequent time step, $t+1$ (DiPasquale and Wheaton, 1996; O'Flaherty, 1996; Waddell, 2000).

$$
\text { if } m \geqslant \theta, v_{t+1}=\lambda v_{t}, \quad \text { else } v_{t+1}=v_{t},
$$

where $0<\lambda<1$.

Household state transition rules. Household automata states are allowed to undergo a transition in two ways. The first is based on the simulation clock for a given run; households progress through life-cycle stages as a simulation ages (Kendig, 1984; Pickvance, 1973), and the duration of their residency in a home changes in a similar way. A second scheme is used to change between household automata states, and this is based on a series of preference functions. These preferences are designed in the tradition of stress-resistance hypotheses (Wolpert, 1966) and largely relate to households' contentment with their own property, the real estate submarket in which the property sits, and the community to which a household belongs. Contentment relates to households' internal states (income, average age, number of children, size, ethnicity, life-cycle stage, duration of residency), the states of the property in which they reside (housing type, tenure, mortgage or rent value, density, number of bedrooms), the condition of the submarket in which the property sits (average property value, maximum property value, minimum property value) and conditions of the community with which they are affiliated (number of households, ethnicity profile, wealth, average age). Contentment is articulated using a series of preference functions: inertia, hedonic property, wealth, and segregation preferences.

An inertia preference is used to generate movers and stayers (Brown and Moore, 1970); it operates across scales and serves to evaluate residential stress (Clark and Cadwaller, 1973; Wolpert, 1966), due to changes internal to the household or to the submarket and community it belongs to [figure 2(b)]. The inertia preference governs whether a household will change to a mobile state or not. It is triggered by three other preference functions: a hedonic property preference, a wealth preference, and a segregation preference.

A hedonic property preference is used to encapsulate households' property needs; real estate is treated as bundles of attributes, each with associated value (Rosen, 1974). For resident households this is used to calculate the value that they place on their property; for relocating households this becomes part of their housing search. Household automata have a preference for housing type and tenure [see Golledge and Stimson (1997) and Clark (1982b) for theoretical background]. In addition, they have a preference for housing price. Type and tenure preferences are formulated, simply, on the basis of the life-cycle state of a household (Clark and Huang, 2003). 'Young' households have a preference to rent apartments, 'middle' households prefer to buy apartments, and 'senior' households prefer to buy houses. The expression of a housing value preference is also specified simply; households will not buy or rent a property that is more expensive than one third of their monthly income. Socioeconomic and cultural preferences operate at a higher scale [figure 2(b)].

Wealth preferences allow household GA to assess whether a neighborhood is too poor for them to enter or remain in, or whether it is too expensive to consider relocating to. This is equivalent to the value orientation discussed by Golledge and Stimson (1997), among others. A submarket GA is regarded as too expensive for a particular household if the average monthly mortgage or rental value of a home $\left(v_{\text {avg }}\right)$ is greater than the household's average income. If the maximum price in the submarket $\left(v_{\max }\right)$ is below a given household's monthly average income $(i)$, then the submarket is 
regarded as being too cheap; if a household's income is lower than the lowest price $\left(v_{\min }\right)$, the submarket is too expensive.

A segregation preference function is used to represent households' likes and dislikes regarding the ethnic profile of residential submarkets. The function is specified in much the same way as Schelling's segregation models (Schelling, 1978) - through the use of tolerance thresholds to specify households' comfort with certain conditions in the submarket, in this case ethnicity. In the simulations to be discussed in later sections, yellow household GA have no preference regarding the ethnic composition of a submarket. Blue household GA exhibit a level of bias in their preferences; they have a preference for submarkets where red household GA form no more than one third of the total population. Red household GA prefer submarkets where they form a majority, above one half of the submarket's residential population.

\subsection{Georeferencing conventions}

A variety of georeferencing conventions are used in the model. At the top level of hierarchy for entities in the model [figure 2(a)], submarkets are georeferenced in terms of their position within a larger urban system. We will consider a single submarket in the simulations to be described. However, multiple submarkets could be georeferenced in a city system by their absolute location within a lattice of submarket automata, each perhaps associated with some distance and/or accessibility to a city center or centers.

Properties are georeferenced directly, with the use of the Cartesian coordinate location of their centroid within the property automata lattice. Household automata are then associated with this point.

Households are georeferenced both directly and indirectly. Settled households are georeferenced to their property using Cartesian coordinates. Relocating households are georeferenced directly - their absolute position within the lattice is registered using Cartesian coordinates. As they search for potential homes they are georeferenced indirectly with respect to the last property they viewed and the next property that they plan to view.

\subsection{Movement rules}

Movement is fashioned as a migration event (Rossi, 1955) in the model. This is fundamentally different to the random cell activation and deactivation mechanisms of other automata-based residential models (O'Sullivan, 2002; Portugali, 2000; Sakoda, 1971; Schelling, 1971). Migration events are composed of independent movement mechanisms - inertia, housing search, displacement, and stopping rules. Through these movements households relocate, migrate, and move within their simulated environments. Those rules animate households through a fixed property environment, but also relate to inertia with respect to shifting real estate and community dynamics.

The decision to relocate, for example, is based on an inertia calculation, as mentioned. This is a fundamental rule in the model; the end result of the rule may be a transition for a household from a fixed state to a mobile state and vice versa.

Once mobile, households engage in a housing search. Modeled households move around the simulated property market by hopping between residential opportunities, one property at a time, evaluating the state conditions they encounter and using these states as input to a preference evaluation decision (Clark, 1970). Their movement through the environment dictates the order in which various property alternatives are evaluated (Clark, 1993).

If households agree on the sale or rental of a property, a displacement rule is initiated, under which the relocating household moves into a property (and changes to a settled state) and the former resident vacates the property (and changes to a relocating state). This rule plays a role in community and submarket formation at a higher scale (van der Vlist et al, 2002); new households alter the overall community 
profile and the price paid for a property may influence average values recorded at a submarket level. Similarly, a change in tenure is reflected in the wider submarket records. In this sense, then, higher level entities progress through states as the conditions in the properties and households within them change. Cross-scale dynamics are thus supported.

Households may enter submarkets for housing search on an exogenous basis, in addition to possessing endogenous relocation decisions. A dedicated rule for entry and exit controls the influx and evacuation of households in this context.

In addition, a stopping rule is used to provide chances for relocating households to terminate their housing search (Clark, 1982b; Clark and Flowerdew, 1982). The opportunity to stop a search presents itself at two scales. A relocating household may elect to abandon a search, at the community and submarket scale, on the basis of its wealth and/or segregation preferences for conditions at that scale. Within a submarket a housing search stops when a relocating household moves into a new home, or when the household has evaluated all available properties but has not been adequately satisfied by any of them.

\subsection{Interaction neighborhoods and rules}

Neighborhood interaction definitions vary in the model, depending on the automata considered. Neighborhoods for submarkets consist of all household and property automata active within a submarket and community. A variety of statistics are generated from these neighborhoods, dynamically, when a simulation is run. The values register as state variables in submarket automata (table 1).

The rule base for neighborhood delineation is based on space and time for household GA. For relocating households the neighborhood initially consists of a submarket as they enter the simulation. Once they have begun to search a submarket, their neighborhood consists of the independent property that they chose to evaluate. At a given transition point after that they register their current location (which may be a property automata), previously viewed property, and next-view property as their neighborhood for interaction.

\subsection{Constraints}

Some constraint parameters are also introduced to the model design to facilitate simulation. First, a growth parameter is included to control the volume of new searching households inputted to the system. A number of relocating households is delivered to the modeled submarket at the start of a simulation run. Similarly, a number of fixed property automata are active in the housing market at the start of a particular simulation run. Dependencies between state variables and preference functions also create a set of constraints. Relocating-household GA may purchase only properties they can afford. Also, relocating households will consider only submarkets that are either affordable or above a certain price threshold. The latter ensures that relatively more affluent households do not relocate to relatively poorer-profile neighborhoods. Furthermore, households are constrained to searches for properties that have enough bedrooms to accommodate their household size.

\subsection{Time and dynamics}

The model is organized to handle time in an event-based fashion (Worboys, 1994). Time is structured into discrete packets of change (Anderson, 2002), nested hierarchically with relation to other such packets. Several clocks operate within the model, each with their own rhythms; modeled entities orchestrate their activities with respect to whichever clock is appropriate.

One of the advantages of an event-based approach in the model is that simulations may incorporate cross-scale dynamics. Lower-scale entities (households, properties) 
may be designed to interact with and react to changing conditions at higher scales (property submarkets, communities of households), as they evolve. For example, as the ethnic profile of a community changes, the household residing in a given property may grow intolerant of the new ethnic profile of the community; having previously been content to stay, the household may choose to relocate.

\section{Simulating residential mobility scenarios}

The simulation operates on an event-based scheme, with events designed to coincide with the various stages that constitute residential-mobility decisions (Clark, 1982b). A typical simulation run proceeds as follows (figure 3 ).

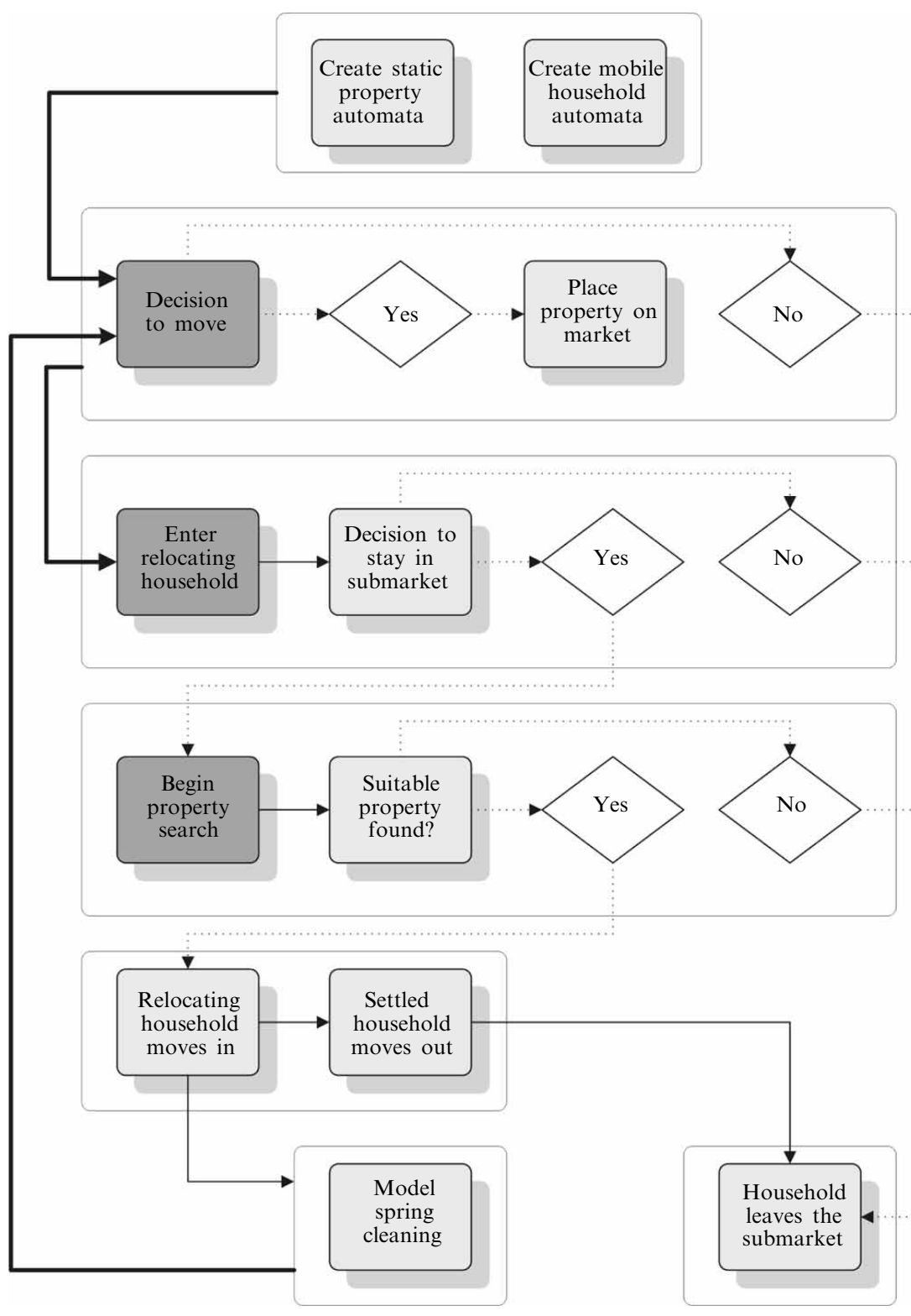

Figure 3. The sequence of events in an iteration of the simulation. 
The first stage in a model run involves the creation of a virtual residential environment. 'Robot' GA are called upon to seed the model with static automata properties. These robot GA enter the model and code attributes into a blank environment, thereby establishing a simulated residential property market. Specific units are rendered active in the model and are assigned heterogeneous property attributes (table 1). Following this, activated property GA are then populated with settled household GA, characterized by heterogeneous life-like attributes.

Once individual property sites have been established and populated, the settled household GA that occupy them call upon their inertia preference function to make a decision about whether to move home or not. This is a multiscale evaluation: household GA look both to their internal household characteristics and also to the attributes of the submarket in which they reside before making a decision [figure 2(b)]. Changes in households' internal states (internal stress) may result in a decision to move - a transition to a new life-cycle stage, a growth or reduction in household size, the arrival of children, etc. At the same time, there may be forces at work at the scale of the submarket (environmental stress) that influence their decisions to relocate or not. These can be initiated endogenously within the system - for example, changes in the socioeconomic profile of a neighborhood through gentrification or neighborhood decline-or could be user specified as exogenous shocks. If a settled household GA decides to move, its property (the individual fixed property GA that the household is associated with) is flagged as being for sale or for rent, as the case may be. This registers in the appropriate state variable for the GA.

At this stage, a relocating household GA is delivered to the model, in search of a new home. Currently, incoming GA are generated synthetically, as randomly-defined or user-defined entities and introduced from the top down; they enter the simulation as a simple feed. Relocating GA hold a set of preferences for their ideal location and home, and must balance these desires within the bounds of what they can afford. Currently, only one relocating household GA populates a given simulation iteration at any instance, although many settled automata are available for it to interact with. First, a relocating household GA looks to the community and submarket to determine if it is suitable for its needs - whether it has the right ethnic profile and whether it is too expensive or too cheap. If the submarket and community are suitable, the relocating household will begin to focus its housing search on individual properties in the submarket. Potential homes are assessed for their suitability to individual households' needs.

The relocating household GA visits active properties randomly, evaluating their characteristics against its preferences. In the simulation described in this section, the evaluation is formulated in a hierarchical fashion. A relocating GA begins the evaluation by checking that the housing type $(h)$ matches the type in its preference set. Next, the relocating household GA evaluates tenure $(r)$, followed by price $(v)$. This is quite a simplistic method for matching preferences with attributes, although it does provide for the weighting of choices in the tradition of discrete choice models (McFadden, 1974).

If a relocating household GA finds a suitable property that matches its preferences and its budget, it trades places with the settled household that occupies the property. The sale (or rental) status of the property is updated to reflect the fact that it is no longer on the market. The new household is moved in, changing status from relocating to settled, and the original household is moved out, again changing status. These adjustments are also registered in the global characteristics of the submarket and community, thereby facilitating change above the scale of an individual. For example, if a red household replaces a blue household, the balance of colors in the community 
will be altered to reflect that; if a household with a very high average income decides to move into a community, the maximum value for average income in the community will be revised to indicate the change.

The model is then put through a series of spring-cleaning exercises before beginning a new iteration. Any relocating GA that have not satisfied their searches after visiting all available properties leave the submarket and disappear from consideration in the model (that is, their stopping rule is triggered by the termination, through failure, of the search in a submarket). If settled households have not sold or rented their property, they may decide to discount the price they are offering the property for in the next iteration of the simulation. By iterating through these events in a sequential fashion, the simulation allows for the evolution of the submarket and community, its population, and the individual properties contained within it. Submarkets could, potentially, go through cycles of decline and gentrification, for example. The socioeconomic composition of the population could also be allowed to cycle through various stages - for example, from a youthful profile to one more characteristic of 'empty-nesters'. The introduction of shocks of various descriptions to the submarket could also allow for the exploration of large-scale responses to things such as an influx of wealthy households, or community response to households of varying sociodemographic backgrounds, from the bottom up. In the next section are presented examples of simulation under such scenarios.

\subsection{Simulation scenarios}

A simulated environment was built, replete with a range of properties, for the purposes of experimenting with two simulation scenarios. The artificial properties were populated with households, forming a synthetic community. A variety of relocating households were introduced. Their housing-search behavior stimulates transition in the submarket and community; incoming households act as a catalyst for change. The influx of new households was tailored to evaluate economic, social, and demographic response in the property environment and household population, across scales from the household and property to the submarket level.

The submarket was constructed as a 250 by 250 unit lattice, in which twenty-nine properties were activated in the market at the start of a simulation (table 2). A community of households was specified randomly within constraining bounds; this yielded a population with ten red households, six yellow households, and nine blue households, with household incomes ranging from $\$ 3000$ to $\$ 15000$ per month (figure 4).

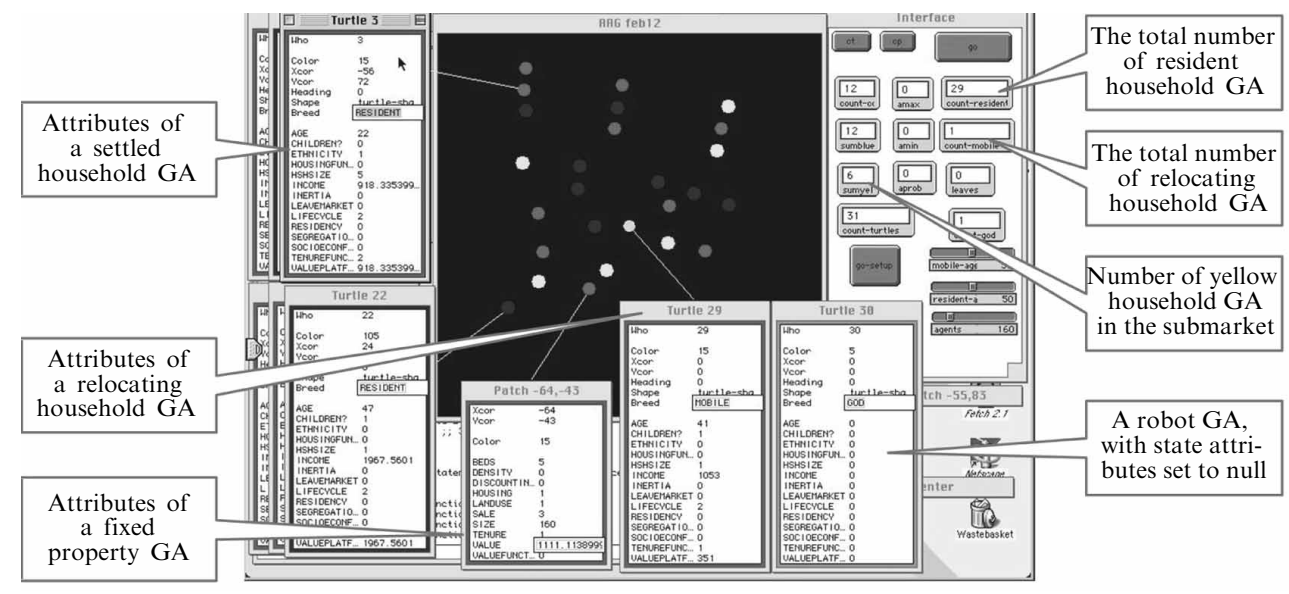

Figure 4. A residential model simulation run, showing geographic automata (GA) attributes. 
Table 2. Initial state variables for a residential mobility simulation. All locations were initially specified as being occupied, and all sites corresponded to residential use with 0 days on the market. Apt and hse refer to apartment and house, respectively.

\begin{tabular}{|c|c|c|c|c|c|c|}
\hline Site location & Housing type & Housing tenure & $\begin{array}{l}\text { Monthly } \\
\text { rent/mortgage }\end{array}$ & Lot size $\left(\mathrm{m}^{2}\right)$ & Density & Bedrooms \\
\hline$(-55,83)$ & apartment & rent & 3000 & 100 & 0.0100 & 2 \\
\hline$(-5,74)$ & apartment & rent & 3300 & 120 & 0.0083 & 2 \\
\hline$(52,63)$ & apartment & rent & 3500 & 140 & 0.0071 & 1 \\
\hline$(-56,72)$ & apartment & rent & 3000 & 90 & 0.0111 & 2 \\
\hline$(-7,62)$ & apartment & rent & 3000 & 100 & 0.0100 & 2 \\
\hline$(48,51)$ & apartment & rent & 3400 & 110 & 0.0091 & 2 \\
\hline$(-55,60)$ & apartment & rent & 5000 & 130 & 0.0077 & 2 \\
\hline$(-9,51)$ & apartment & rent & 5200 & 140 & 0.0071 & 2 \\
\hline$(46,40)$ & apartment & rent & 6200 & 160 & 0.0063 & 3 \\
\hline$(-57,33)$ & house & own & 3000 & 100 & 0.0100 & 4 \\
\hline$(-27,31)$ & house & own & 4300 & 110 & 0.0091 & 3 \\
\hline$(15,23)$ & house & own & 3000 & 85 & 0.0118 & 4 \\
\hline$(34,18)$ & house & own & 3000 & 80 & 0.0125 & 2 \\
\hline$(52,11)$ & house & own & 5000 & 100 & 0.0100 & 4 \\
\hline$(-27,19)$ & house & own & 3000 & 90 & 0.0111 & 3 \\
\hline$(21,10)$ & house & own & 2500 & 90 & 0.0111 & 4 \\
\hline$(-48,6)$ & house & own & 3000 & 200 & 0.0050 & 4 \\
\hline$(-18,1)$ & house & own & 5500 & 210 & 0.0048 & 3 \\
\hline$(20,19)$ & house & own & 5000 & 175 & 0.0057 & 3 \\
\hline$(40,14)$ & house & own & 5000 & 180 & 0.0056 & 4 \\
\hline$(46,-14)$ & house & rent & 2000 & 50 & 0.0200 & 5 \\
\hline$(-12,14)$ & house & rent & 5500 & 100 & 0.0100 & 2 \\
\hline$(24,-44)$ & house & own & 6500 & 400 & 0.0025 & 3 \\
\hline$(73,-65)$ & house & own & 7500 & 400 & 0.0025 & 6 \\
\hline$(-21,-33)$ & house & own & 6500 & 110 & 0.0091 & 4 \\
\hline$(-48,-30)$ & house & own & 10000 & 350 & 0.0029 & 3 \\
\hline$(-64,-43)$ & house & rent & 4000 & 160 & 0.0063 & 5 \\
\hline$(13,-72)$ & house & own & 5600 & 100 & 0.0100 & 3 \\
\hline$(-55,-76)$ & house & own & 6000 & 550 & 0.0018 & 6 \\
\hline
\end{tabular}




\subsubsection{An economic scenario}

The goal of the simulation scenarios is to test the resilience of the systems to change from the bottom up. For the purposes of evaluating economic dynamics, households with very low average incomes were introduced to the simulation, as were households with very high average incomes, each household performing a search on the basis of its hedonic preferences. Both sets of households evaluated the income profile of the submarket and promptly terminated their search, as their preference rules for value platform determined. Next, a household with a price preference that was within an acceptable range was introduced. The household elected to begin a search within the submarket. However, its preference for housing type was not satisfied within that price range, and it left the submarket after evaluating all available properties.

The discounting function, designed to lower the price of a property in the simulation-if there was insufficient demand for it-provided only minor changes in the economic dynamics of submarkets. This is because budgets for relocating households were based on their income in the model; this ensured that very affluent households did not buy or rent very cheap houses, and vice versa. So, small reductions in property values did very little to permit access to submarkets for lower income groups, particularly because incoming relocating households looked to the average value of the submarket before deciding whether to continue a search. This is what happens in the real world; gentrification and decline are slow processes, and are likely to be more dependent on factors such as crime, social problems, and the quality of recreation and retail opportunities in the submarket - factors not represented in this model. However, the price profile of the submarket was also quite resilient to changes in the internal dynamics within the households that form the submarket. Adjusting the average income of individual settled households did not affect the price of property in the region, because the price at which a household sold or rented a property was not linked to its income in the model; at this scale, price was purely demand driven.

The resilience of the simulation to these changes is consistent with situations in residential submarkets in many urban areas in the United States. The sorts of phenomena observed in these experiments are indicative of lock-out situations in wealthy suburbs, whereby lower income groups are barred entry to suburban areas because properties there are not affordable (for discussion in US contexts, see Cronin, 1982; Farley and Frey, 1994; Farley et al, 1978; Galster, 1991; Knox, 1989). Even if affordable housing is constructed in these areas, lower income groups may still be excluded by minimum lot requirements_zoning codes that place a minimum size on residential lots. For example, a minimum lot ordinance calling for half-acre lots raises the overall price of real estate, even if the structure price of a property is relatively low, simply because the land component of the price remains high.

\subsubsection{A sociodemographic scenario}

A series of targeted sociodemographic experiments were then performed with the model. An experiment was run to investigate life-cycle dynamics in the model. Relocating households with lower-than-average and higher-than-average ages were introduced to the community. As these households began to occupy properties in the simulation, so the demographic profile of the submarket began to change accordingly. Similarly, experiments were performed whereby household-size variables were changed for particular settled households. If the increase in household size surpassed the number of bedrooms in their home, they placed their property on the market for sale or rent. In this sense, natural demographics and life-cycle transition act as a catalyst or the impetus for submarket and community change. 
Similarly, changing social dynamics act as drivers of residential transition in another experiment. A stream of rich households of varying colors was introduced to the simulation, with incomes within the acceptable bounds for property prices in the submarket. As relocating households found suitable properties to buy or rent, they moved into the submarket, displacing the previous residents. When the incoming household displaced a household of a different color, the ethnic profile of the submarket altered to reflect that change. Slowly, the balance of ethnicity in the submarket began to change. This prompted two effects. The incoming households began to react differently, rejecting the submarket, depending on their particular preference for ethnic profile. Also, the internal dynamics of the submarket began to change. As the ethnic balance of the submarket altered, the 'stressors' of previously settled household GA were triggered by the shift in balance, and they began to place homes on the market. By varying the ethnicity of incoming relocating household GA, it was possible to shift the ethnic balance of the submarket from mixed conditions to dominance in each of the colors, and from segregated to mixed conditions. Overall, a phenomenon of sociospatial segregation is mimicked akin to the classic Sakoda (1971) and Schelling (1978) models. However, the model used here diverges considerably from the simple formulation of those examples. The behavioral basis allows for investigation with the household-specific and property-specific components responsible for driving segregation, as well as the dynamics of those interactions across scales. This is a topic of my ongoing research.

\section{Conclusions}

The purpose of this paper is to introduce a new approach to modeling residential mobility, on the basis of GA. I believe the technique to be useful for such purposes and have demonstrated its use with references to construction of a richly specified model of residential mobility.

The model focuses on residential location dynamics, specified at an 'atomic' scale - the description of heterogeneous individual households and properties, which are dynamically active within a larger community and residential submarket. The switch to a local scale continues within the model parameters, and is manifest in the fidelity of entities within the model. The model is built around a GA methodology.

Individual-scale entities are described with life-like state variables. However, their independent decisionmaking behaviors in space are also specified, with transition rules designed to represent preferences for housing, house markets, and communities.

The model is valuable as a 'what-if' tool for experimenting with scenarios relating to residential dynamics. I have illustrated the application of the model for these purposes in the context of economic and sociodemographic scenarios. A series of experiments run with the model were described, which focused on an evaluation of the sensitivity of the simulated community or submarket to changes in its economic, demographic, and ethnic profile, as evolved from the bottom up. These scenarios made use of the geographic functionality of the model. In particular, the spatial functionality of the framework facilitated the design of simulations that closely represent residential property markets and communities of residents and their dynamics. Entities of varying sizes could be designed, and nested neatly with respect to their position in a cross-scale and dynamic hierarchy. Varying neighborhood functions were also facilitated, as were direct and indirect georeferencing conventions. Also, the ability to accommodate migration rules was quite significant.

We can draw some conclusions about the general value of the methodology for modeling residential mobility, stemming from its geography-specific formulation. To a certain degree the approach demonstrated in this paper might be regarded as breathing 
fresh life into the residential-mobility-modeling literature. The infusion of spatially explicit functionality and mechanisms, in particular, is significant. We can draw other conclusions.

The first refers to cross-scale dynamics. As the model demonstrates, GA enable the organization of processes and phenomena at appropriate spatial scales, with functionality - states, state transition rules, georeferencing conventions, movement rules, neighborhood relationships, and neighborhood rules-needed at that level of detail. Larger-scale patterns and behaviors evolve across scale barriers, from a foundation lower down in the hierarchy. Lower level phenomena may be designed to respond to conditions at a higher scale, organically and dynamically, as they emerge over the course of a simulation run.

Spatial interaction is a second consideration. Spatial interaction is flexible under GA and may be richly expressed through movement-mediated behavior. Interaction across scales is supported, as noted, and the emergence and capture of novel patterns and processes is possible. There may be any number and form of relationships between entities in a GA model. Direct and indirect dependencies and associations have been demonstrated in this paper, but others may be formulated, as Voronoi adjacencies, entity-relationship links, etc.

The third consideration relates to ontology based on fixture in space. The notion is facilitated in the GA methodology and is incorporated into the model described in this paper. The idea is useful for delineating entities and formulating their behaviors and interactions in a model. Considered cursorily, it helps to distinguish between cellular and agent automata. The functionality of both can be subsumed by GA, and this may help to avoid confusion-for example, when CA cells are made to mutate magically like bacteria in an agar environment - in cases in which such behavior is not appropriate, say in the context of property and real estate transition. More profoundly, ontology based on fixture in space allows for the specification of geographic entities, flexibly endowed with geographic functionality. The methodology is not constrained, for example, by static neighborhood filters that deny action at a distance; mobile and fixed entities may be defined independently and may be made to interact (or not) in dynamic spaces. This has important implications for the potential use of GA tools for theory building or theory testing. The questions that might be asked and answered with GA are not constrained by the methodology underpinning the tools they might be used to build.

\section{References}

Anderson J, 2002, "Providing a broad spectrum of agents in spatially explicit simulation models: the Gensim approach", in Integrating Geographic Information Systems and Agent-based Modeling Techniques for Simulating Social and Ecological Processes Ed. H R Gimblett (Oxford University Press, Oxford) pp $21-55$

Batty M, 1997, "Cellular automata and urban form: a primer" Journal of the American Planning Association $63266-274$

Batty M, 2001, "Polynucleated urban landscapes" Urban Studies 38 635-655

Benenson I, 1998, "Multi-agent simulations of residential dynamics in the city" Computers, Environment and Urban Systems 22 25-42

Benenson I, Torrens P M, 2004 Geosimulation: Automata-based Modeling of Urban Phenomena (John Wiley, New York)

Benenson I, Torrens P M, 2005, "A minimal prototype for integrating GIS and geographic simulation through geographic automata systems", in GeoDynamics Ed. F Wu (CRC Press, Boca Raton, FL) pp $347-369$

Benenson I, Omer I, Hatna E, 2002, "Entity-based modeling of urban residential dynamics: the case of Yaffo, Tel Aviv" Environment and Planning B: Planning and Design 29491 - 512

Benenson I, Aronovich S, Noam S, 2005, "Let's talk objects: generic methodology for urban high-resolution simulation" Computers, Environment and Urban Systems 29425 - 453 
Box P, 2001, "Spatial units as agents: making the landscape an equal player in agent-based simulations", in Integrating Geographic Information Systems and Agent-based Modeling Techniques for Simulating Social and Ecological Processes Ed. H R Gimblett (Oxford University Press, Oxford) pp 59-83

Brown L A, Moore E A, 1970, "The intra-urban migration process: a perspective" Geografiska Annaler 52B $1-13$

Clark W A V, 1970, "Measurement and explanation in intra-urban mobility" Tijdschrift voor Economische en Sociale Geografie $6149-57$

Clark W A V, 1982a, "Recent research on migration and mobility: a review and interpretation" Progress and Planning $181-56$

Clark W A V (Ed.), 1982b Modelling Housing Market Search (Croom Helm, London)

Clark W A V, 1993, "Search and choice in urban housing markets", in Behavior and Environment: Psychological and Geographical Approaches Eds T Gärling, R G Golledge (Elsevier, Amsterdam) pp $298-316$

Clark W A V, Cadwaller M, 1973, "Locational stress and residential mobility” Environment and Behavior $529-41$

Clark W A V, Flowerdew R, 1982, "A review of search models and their application to search in the housing market", in Modelling Housing Market Search Ed. W A V Clark (Croom Helm, London) pp 4-29

Clark W A V, Huang Y, 2003, "The life course and residential mobility in British housing markets" Environment and Planning A $35323-339$

Cronin F J, 1982, "Racial differences in the search for housing", in Modelling Housing Market Search Ed. W A V Clark (Croom Helm, London) pp 81 - 106

DiPasquale D, Wheaton W, 1996 Urban Economics and Real Estate Markets (Prentice-Hall, Englewood Cliffs, NJ)

Epstein J M, 1999, "Agent-based computational models and generative social science" Complexity $441-60$

Faith J, 1998, "Why gliders don't exist: anti-reductionism and emergence", in Artificial Life VI: Proceedings of the Sixth International Conference on Artificial Life Ed. C Adami (MIT Press, Cambridge, MA)

Farley R, Frey W H, 1994, "Changes in the segregation of whites from blacks in the 1980s: small steps toward a more integrated society" American Sociological Review 5923 -45

Farley R, Schuman H, Binachi S, Colasanto D, Hatchett S, 1978, "Chocolate city, vanilla suburbs: will the trend toward racially separate communities continue?" Social Science Research $7319-344$

Ferber J, 1999 Multi-agent Systems: An Introduction to Distributed Artificial Intelligence (Addison-Wesley, Harlow, Essex)

Galster G, 1991, "Black suburbanization: has it changed the relative location of the races?" Urban Affairs Quarterly 26621 - 628

Gardner M, 1970, "The fantastic combinations of John Conway's new solitaire game 'Life" " Scientific American 223120 - 123

Gardner M, 1971, "Mathematical games: on cellular automata, self-reproduction, the garden of Eden, and the game 'life" "Scientific American 224112 - 117

Golledge R, R J Stimson, 1997 Spatial Behavior: A Geographic Perspective (Guilford Press, New York)

Kendig H, 1984, "Housing careers, life cycle, and residential mobility: implications for the housing market" Urban Studies 4271 - 283

Knox P, 1989, "The vulnerable, the disadvantaged, and the victimized: who they are and where they live", in Social Problems and the City Eds D T Herbert, D M Smith (Oxford University Press, Oxford)

Kohler T A, Gumerman G, 2001 Dynamics in Human and Primate Societies (Oxford University Press, Oxford)

McFadden D, 1974, "Conditional logit analysis of qualitative choice behavior", in Frontiers in Econometrics Ed. P Zarembka (Academic Press, New York)

O'Flaherty B, 1996 Making Room: The Economics of Homelessness (Harvard University Press, Cambridge, MA)

O'Sullivan D, 2002, “Toward micro-scale spatial modeling of gentrification” Journal of Geographical Systems $4251-274$

Peckham J, MacKellar B, Doberty M, 1995, "Data models for extensible support of explicit relationships in design databases" The VLDB Journal $4157-191$ 
Pickvance G C, 1973, "Life-cycle, housing tenure and intra-urban residential mobility: a causal model" American Sociological Review $21279-297$

Portugali J, 2000 Self-organization and the City (Springer, Berlin)

Portugali J, Benenson I, Omer I, 1994, "Socio-spatial residential dynamics: stability and instability within a self-organized city" Geographical Analysis 26321 - 340

Portugali J, Benenson I, Omer I, 1997, "Spatial cognitive dissonance and sociospatial emergence in a self-organizing city" Environment and Planning B: Planning and Design 24 263-285

Rosen S, 1974, "Hedonic prices and implicit markets: product differentiation in pure competition" Journal of Political Economy 82 34-35

Rossi P H, 1955 Why Families Move: A Study in the Social Psychology of Urban Residential Mobility (Free Press, Glencoe, IL)

Russell S, Norvig, 1995 Artificial Intelligence: A Modern Approach (Prentice-Hall, Englewood Cliffs, NJ)

Sakoda J M, 1971, "The checkerboard model of social interaction" Journal of Mathematical Sociology $1119-132$

Schelling T C, 1969, "Models of segregation" American Economic Review 59488 - 493

Schelling T C, 1971, "Dynamic models of segregation” Journal of Mathematical Sociology 1143 - 186

Schelling T C, 1974, "On the ecology of micro-motives", in The Corporate Society Ed. R Marris (Macmillan, London)

Schelling T C, 1978 Micromotives and Macrobehavior (W W Norton, New York)

Schweitzer F, 2003 Brownian Agents and Active Particles (Springer, Berlin)

Shi W, Pang M Y C, 2000, "Development of Voronoi-based cellular automata - an integrated dynamic model for geographical information systems" International Journal of Geographical Information Science 14 455-474

Sipper M, 1997 Evolution of Parallel Cellular Machines: The Cellular Programming Approach, Lecture Notes in Computer Science 1194 (Springer, Berlin)

Speare A, Goldstein S, Frey W H, 1975 Residential Mobility, Migration and Metropolitan Change (Ballinger, Cambridge, MA)

Stefansson B, 1997, "Swarm: an object-oriented simulation platform applied to markets and organizations", in Evolutionary Programming 6 Eds P Angeline, R Reynolds, J McDonnell, R Eberhart (Springer, New York)

Tesfatsion L, 2002, "Agent-based computational economics: growing economies from the bottom up", Department of Economics, Iowa State University, Ames

Torrens P M, 2005, "Geosimulation approaches to traffic modeling", in Transport Geography and Spatial Systems Eds P Stopher, K Button, K Haynes, D Hensher (Pergamon, London)

Torrens P M, Benenson I, 2005, "Geographic automata systems" International Journal of Geographical Information Science $19385-412$

van der Vlist A J, Gorter C, Nijkamp P, Rietveld P, 2002, "Residential mobility and local housing-market differences" Environment and Planning A $341147-1164$

Waddell P A, 2000, "A behavioural simulation model for metropolitan policy analysis and planning: residential location and housing market components of UrbanSim" Environment and Planning B: Planning and Design $27247-263$

Wolpert J, 1965, "Behavioral aspects of the decision to migrate" Papers and Proceedings of the Regional Science Association 15159 - 169

Wolpert J, 1966, "Migration as an adjustment to environmental stress" Journal of Social Issues $2292-102$

Woolridge M J, Jennings N R, 1995, "Intelligent agents: theory and practice" Knowledge Engineering Review 10115 - 152

Worboys M, 1994, "A unified model of spatial and temporal information" Computer Journal 37 $26-34$

Wu F, Li S, 2004, "Theme issue: residential mobility and housing choice in China" Environment and Planning A $361-189$ 